\title{
Ultrastructure of endothelial cells of fetal capillaries of placentae of women with pregnancy induced hypertension
}

\author{
*Sujatha S. Salgado1, Preethika Angunawela², A. de Tissera ${ }^{3}$, Jayantha Sirisena"
}

The Ceylon Journal of Medical Science 2006; 49: 13-20

\begin{abstract}
The present study aimed to compare the ultra structural features of endothelial cells of fetal terminal capillaries of placentae from hypertensive $(\mathrm{n}=10)$ and non-hypertensive $(\mathrm{n}=10)$ pregnancies.
\end{abstract}

The ultrastructural features of endothelial cells of terminal capillaries from the fetal surface of each placenta were examined under the electron microscope.

Micrographs of 8 different endothelial cells from each placenta were prepared for evaluation by stereological analysis using a coherent double lattice test system. The volume fractions of different organelles and cytosol in endothelial cells of placental fetal capillaries of both hypertensive and non-hypertensive pregnancies were determined by counting the number of grid intersection points falling on the endothelial cell cytoplasm, mitochondria, rough endoplasmic reticulum and glycogen deposits.

Ultrastructural changes were noted in endothelial cells of capillaries of placentae of mothers with pregnancy induced hypertension. Statistical analysis showed that the volume fractions of the organelles and the amount of cytoplasm of endothelial cells of placentae from hypertensive pregnancies were significantly higher than those from non-hypertensive pregnancies.

There was a significant increase in some of the organelles and the capacity of cytoplasm of endothelial cells of placentae from hypertensive pregnancies when compared to normotensive pregnancies. These findings may be attributed to compensatory mechanisms operating as a response to hypoxia caused by reduced maternal blood flow to the placenta.

Key words: capillaries, endothelial cells, hypertension, organelles, electron micrographs, stereological estimation.

\section{Introduction}

The human placental fetal capillaries form an important component involved in the exchange of substances between the mother and the fetus. Ultrastructurally the placental capillary wall consists of a single layer of endothelial cells, a basal lamina and occasional pericytes. The endothelium is of

1. Department of Anatomy, Faculty of Medicine, University of Kelaniya, Thalagolla Road, Ragama.

2. Department of Pathology, Faculty of Medicine, University of Colombo, Kynsey Road, Colombo 8.

3. Medical Research Institute, Colombo 8.

4. Department of Obstetrics E Gynaecology, Faculty of Medicine, University of Kelaniya, Thalagolla Road, Ragama.

* Author for correspondence. Received on April 24, 2006 and revised version accepted on August 27, 2006. 
the continuous type which is connected by tight junctions $(1,2)$. The placental fetal capillary endothelial cells are metabolically active as demonstrated by the presence of mitochondria, rough endoplasmic reticulum and glycogen deposits. Their importance in fetomaternal transfer of substances is shown by the abundance of pinocytotic vesicles.

Many theories have been put forward to explain the pathogenesis of pregnancy induced hypertension. Abnormal placentation and reduced uteroplacental blood flow are associated with pregnancy induced hypertension. Normotensive pregnancy is associated with increased blood flow in the uteroplacental circulation in parallel with the developing fetoplacental circulation. On the maternal side the uteroplacental bed is progressively transformed from a high pressure low flow system to a low pressure high flow system in order to meet the requirements of both the placenta and the developing fetus. On the fetal side, normally low fetoplacental perfusion pressure occurs as a result of the release of vasoactive substances and the anatomical development of the distal branches of the fetal villous tree $(3,4)$. Extensive ultrastructural endothelial cell injury has been found in placental bed specimens in pre-eclampsia. It has been proposed that pre-eclampsia is a two stage placental disease. The first stage being attributed to the abnormalities in the normal processes which affect uteroplacental blood supply. The second stage is believed to encompass the effects of the resulting placental ischaemia on the fetal and maternal circulation (5). In pre-eclampsia the microvilli of the syncytiotrophoblast are abnormally shaped and there are focal areas of necrosis (6). It was shown that the isolated syncytiotrophoblast microvillus membrane could inhibit endothelial cell growth and sloughed off microvilli may be responsible for the maternal syndrome of pre-eclampsia due to the resulting endothelial cell damage (7).
Endothelial cells subserve complex functions to mediate immune and inflammatory responses, maintain integrity of the vascular compartment, prevent intravascular coagulation and modify contractile responses of the underlying smooth muscle cells (8). As data have accumulated it has become increasingly evident that the insult to the endothelium is neither toxicity nor nonspecific injury but rather can better be characterized as endothelial activation (9). An imbalance in placental prostacyclin and thromboxane production has been shown to occur in pre-eclampsia. Dysfunctional endothelial cells produce less prostacyclin and activate platelets that subsequently release thromboxane. This situation could profoundly and adversely affect the uteroplacental blood flow, grow th of the fetus, and maternal vascular changes (10). The most consistent morphological abnormality in pre-eclamptic women is the renal lesion termed glomerular endotheliosis. In this instance glomerular capillary endothelial cells are engorged with intracellular inclusions. The clinical features of preeclampsia are edema and the glomerular capillary protein leak associated with deranged renal endothelial transport function. Umbilical cord arteries from infants of pre-eclamptic women have been reported to demonstrate endothelial cell disruption, which is not present in similar vessels of infants of normal women (11). Therefore the present study was undertaken to study the ultrastructural features of fetal endothelial cells of placentae of hypertensive and nonhypertensive pregnancies.

\section{Material and Methods}

The study sample consisted of the mothers admitted for delivery to the antenatal nonpaying wards of the Teaching Hospital, Ragama during the period from May 1995 to May 1996. Ten non-hypertensive primipara pregnant mothers admitted for 
delivery after 37 completed weeks of gestational age who had uncomplicated pregnancies and delivered normal healthy babies weighing at least 2500 grams, between 37 to 41 weeks of gestation were selected at random and 10 pregnant mothers who had a blood pressure of $140 / 90 \mathrm{mmHg}$ or more on admission, persisting for the next 24 hours and who had developed hypertension after the 20 th week of gestation were included in the study sample.

Hypertensive mothers had their babies delivered between 36-40 weeks of gestation. Mothers who were diagnosed as having chronic hypertension or had a history of hypertension before 20 weeks of gestation were excluded.

Immediately after delivery placental membranes and umbilical cord were removed and the weight and the diameters of the placentae were measured using a scale and a fiber tape respectively. Subsequently a macroscopic examination was done looking for infarcted areas and other abnormalities. Placental tissue of about 2 $\mathrm{mm}$. in size was taken from the central and peripheral areas of the fetal surface of the placenta and fixed in $2 \%$ gluteraldehyde overnight and post-fixed in $1 \%$ osmium tetroxide for one hour at $4{ }^{\circ} \mathrm{C}$.

The test material was then dehydrated in ascending concentrations of alcohol, cleared with propylene oxide and then embedded in Epoxy resin. Ultrathin sections $(70 \mathrm{~nm})$ of these pieces of placenta were stained with uranyl acetate and lead citrate. They were examined with a Joel 1200 electron microscope. Endothelial cells of the capillaries of terminal villi were selected systematically with a random start point using the corners of the supporting copper grid square as a sampling reference. From each placenta, micrographs of eight different endothelial cells were prepared at a magnification of
5000 to provide information of statistical validity (12). Micrographs were printed from the negatives at a final magnification of $25,000(5000 \times 5)$.

\section{Stereological Estimation}

A coherent double lattice test system printed on a clear plastic was used for the stereological analysis of the electron micrographs. The coarse grid pattern $25 \mathrm{~mm}$. spacing of the double lattice was used to determine the number of grid intersection points falling on endothelial cell cytoplasm, normal mitochondria, rough endoplasmic reticulum (rER) and glycogen deposits. The area used for this consisted of 24 points on the coarse grid pattern. The volume fractions $(\mathrm{Vv})$ of the different organelles of endothelial cells of placentae from hypertensive and non-hypertensive pregnancies were then obtained.

Volume fraction of component a (Vvac) is the number of component a contained within the (endothelial cell cytoplasm) volume $c$ which is calculated by $\mathrm{Vvac}=\mathrm{Pa} / \mathrm{Pc}$ $\mathrm{Pa}=$ No. of test points falling on the " $a$ " component

$\mathrm{Pc}=$ No. of test points falling on the volume "c"(13).

\section{Statistical analysis}

Student's $t$ test was carried out to find out whether there was a significant difference in the volume fractions of the organelles and the cytoplasm of placental fetal capillary endothelial cells of the non-hypertensive and the hypertensive groups. Epi-info statistical package was used in the analysis.

\section{Results}

Two mothers were on medication for hypertension. Three neonates were below the average birth weight of 2500 grams. 
The preservation of mitochondrial structure of the endothelial cells indicated that the quality of fixation was good. The section thickness was in the silver/gold color interference range. There was a preponderance of organelles around the nuclear region. In most of the terminal capillaries of placentae of hypertensive mothers, the endothelial cell thickness was increased. It was also observed that the number of mitochondria and rough endoplasmic reticulum in those endothelial cells were increased. Mitochondrial vacuolation and distorted mitochondrial architecture were noted in some endothelial cells of capillaries of placentae of hypertensive mothers (Figures 1,2).

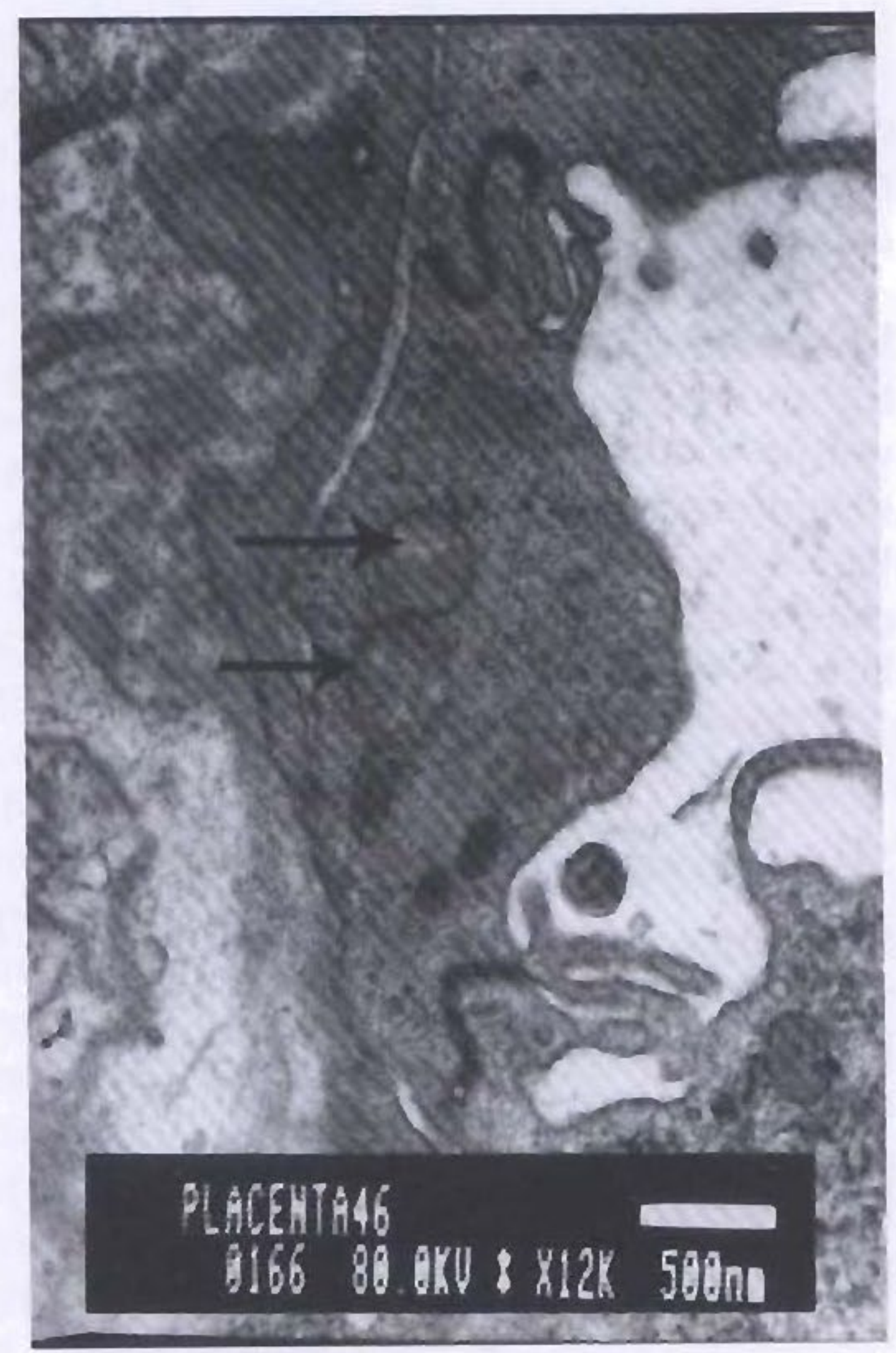

Figure 1. Electron micrograph of a terminal chorionic villous capillary of a placenta of a non-hypertensive mother showing an endothelial cell with normal mitochondria indicated by arrows $(\times 25,000)$.

\section{Quantitative Estimation}

The volume fractions of endothelial cell cytoplasm $(\mathrm{Vv}=$ volume fraction of cytoplasm per unit volume of tissue) and cell components $(\mathrm{VV}=$ volume fraction of structure per unit volume of cytoplasm) of placental capillaries of non-hypertensive and hypertensive groups were calculated (Table 1). Statistically significant increase in volume fractions of endothelial cell cytoplasm, mitochondria, rough endoplasmic reticulum and glycogen deposits were seen in capillary endothelial cells of placentae of hypertensive group than those of non-hypertensive group (Table 2, Figure 3).

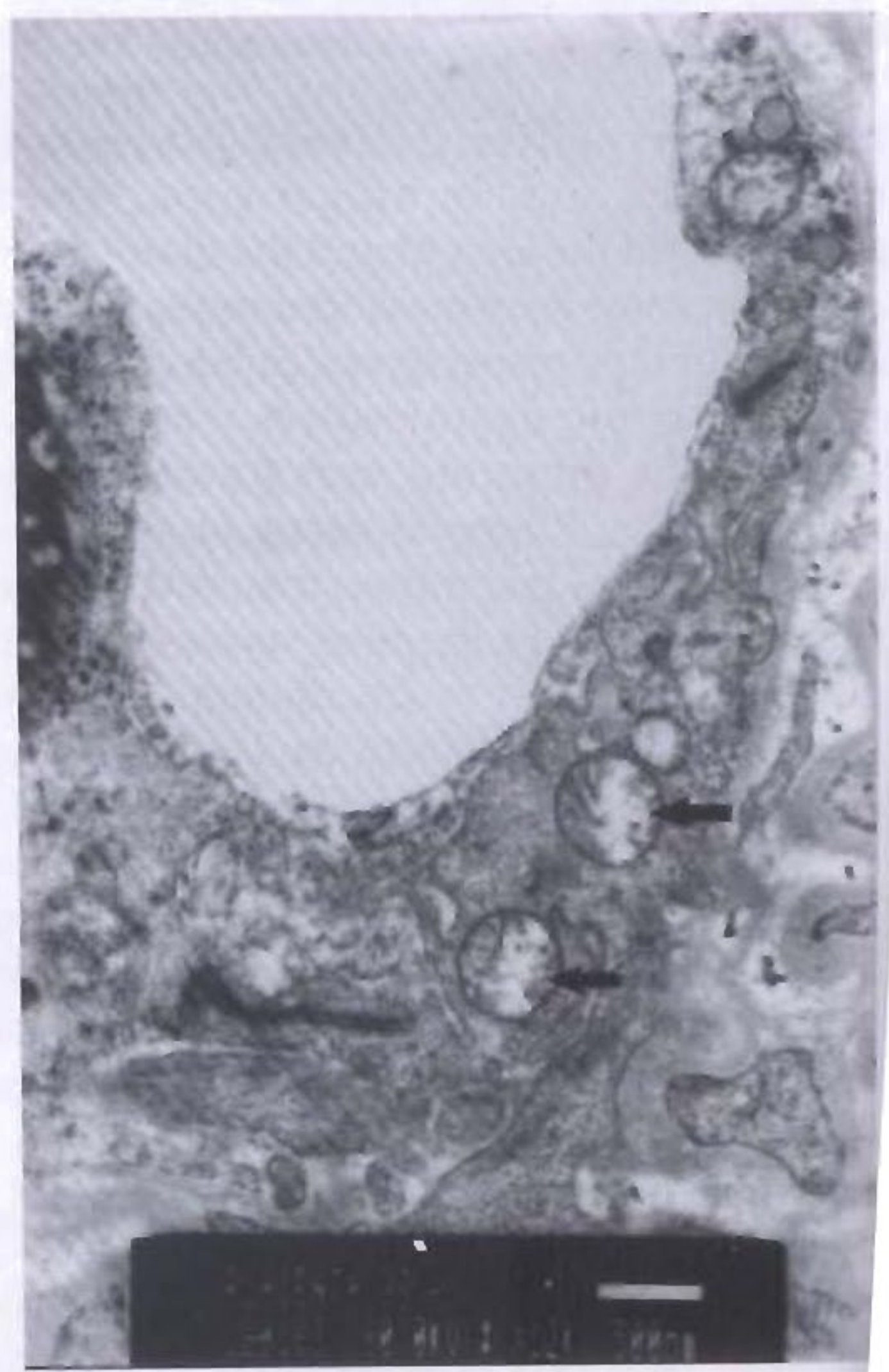

Figure 2. Electron micrograph of a termina chorionic villous capillary of a placenta o a hypertensive mother showing as endothelial cells with vacuolated mitochor dria indicated by arrows $(\times 25,000)$. 
Table 1. The volume fractions (Vv) of capillary endothelial cell components of placentae of non-hypertensive and hypertensive mothers

\begin{tabular}{|c|c|c|c|c|c|c|c|c|c|c|}
\hline \multirow[t]{2}{*}{$\begin{array}{l}\text { No.of } \\
\text { Placenta }\end{array}$} & \multicolumn{2}{|c|}{ No.of Micrographs } & \multicolumn{2}{|c|}{$\begin{array}{l}\text { Volume fraction (Vv) } \\
\text { Cytoplasm }\end{array}$} & \multicolumn{2}{|c|}{$\begin{array}{l}\text { Volume fraction (Vv) } \\
\text { Mitochondria }\end{array}$} & \multicolumn{2}{|c|}{$\begin{array}{l}\text { Volume fraction (Vv) } \\
\text { rER }\end{array}$} & \multicolumn{2}{|c|}{$\begin{array}{l}\text { Volume fraction (Vv) } \\
\text { Glycogen deposits }\end{array}$} \\
\hline & Normal & Hypertensive & Normal & Hypertensive & Normal & Hypertensive & Normal & Hypertensive & Normal & Hypertensive \\
\hline 1 & 8 & 8 & 0.438 & 0.646 & 0.071 & 0.113 & 0.059 & 0.097 & 0.095 & 0.113 \\
\hline 2 & 8 & 8 & 0.250 & 0.563 & 0.041 & 0.111 & 0.041 & 0.130 & 0.103 & 0.103 \\
\hline 3 & 8 & 8 & 0.438 & 0.416 & 0.071 & 0.075 & 0.059 & 0.125 & 0.047 & 0.055 \\
\hline 4 & 8 & 8 & 0.333 & 0.313 & 0.046 & 0.100 & 0.046 & 0.100 & 0.062 & 0.063 \\
\hline 5 & 8 & 8 & 0.438 & 0.687 & 0.059 & 0.090 & 0.059 & 0.045 & 0.071 & 0.075 \\
\hline 6 & 8 & 8 & 0.438 & 0.438 & 0.083 & 0.047 & 0.059 & 0.025 & 0.059 & 0.055 \\
\hline 7 & 8 & 8 & 0.479 & 0.520 & 0.042 & 0.060 & 0.054 & 0.080 & 0.043 & 0.041 \\
\hline 8 & 8 & 8 & 0.250 & 0.750 & 0.041 & 0.111 & 0.041 & 0.083 & 0.103 & 0.103 \\
\hline 9 & 8 & 8 & 0.438 & 0.417 & 0.071 & 0.125 & 0.035 & 0.075 & 0.036 & 0.037 \\
\hline 10 & 8 & 8 & 0.333 & 0.625 & 0.031 & 0.100 & 0.031 & 0.083 & 0.048 & 0.052 \\
\hline
\end{tabular}

$\mathrm{rER}=$ Rough endoplasmic reticulum 
Table 2. Comparison of volume fractions $(\mathrm{Vv})$ of endothelial cell cytoplasm and organelles of capillaries of placentae of non-hypertensive and hypertensive mothers

\begin{tabular}{lllll}
\hline & $\begin{array}{l}\mathrm{V}_{v} \text { Non-hypertensive } \\
\mathrm{n}=10 \\
\text { Mean (SD) }\end{array}$ & $\begin{array}{l}\mathrm{V}_{\mathrm{v} \text { Hypertensive }} \mathrm{n}=10 \\
\text { Mean }(\mathrm{SD})\end{array}$ & t Value & $\mathrm{P}$ Value \\
\hline Cytoplasm & $0.372(0.083)$ & $0.538(0.140)$ & 3.653 & $\mathrm{P}<0.001^{*}$ \\
Mitochondria & $0.056(0.018)$ & $0.093(0.031)$ & 3.272 & $\mathrm{P}<0.001^{*}$ \\
rER & $0.048(0.010)$ & $0.084(0.032)$ & 3.600 & $\mathrm{P}<0.001^{*}$ \\
Glycogen & $0.065(0.025)$ & $0.115(0.038)$ & 3.571 & $\mathrm{P}<0.001^{*}$ \\
\hline
\end{tabular}

$\mathrm{rER}=$ Rough Endoplasmic Reticulum * Highly significant

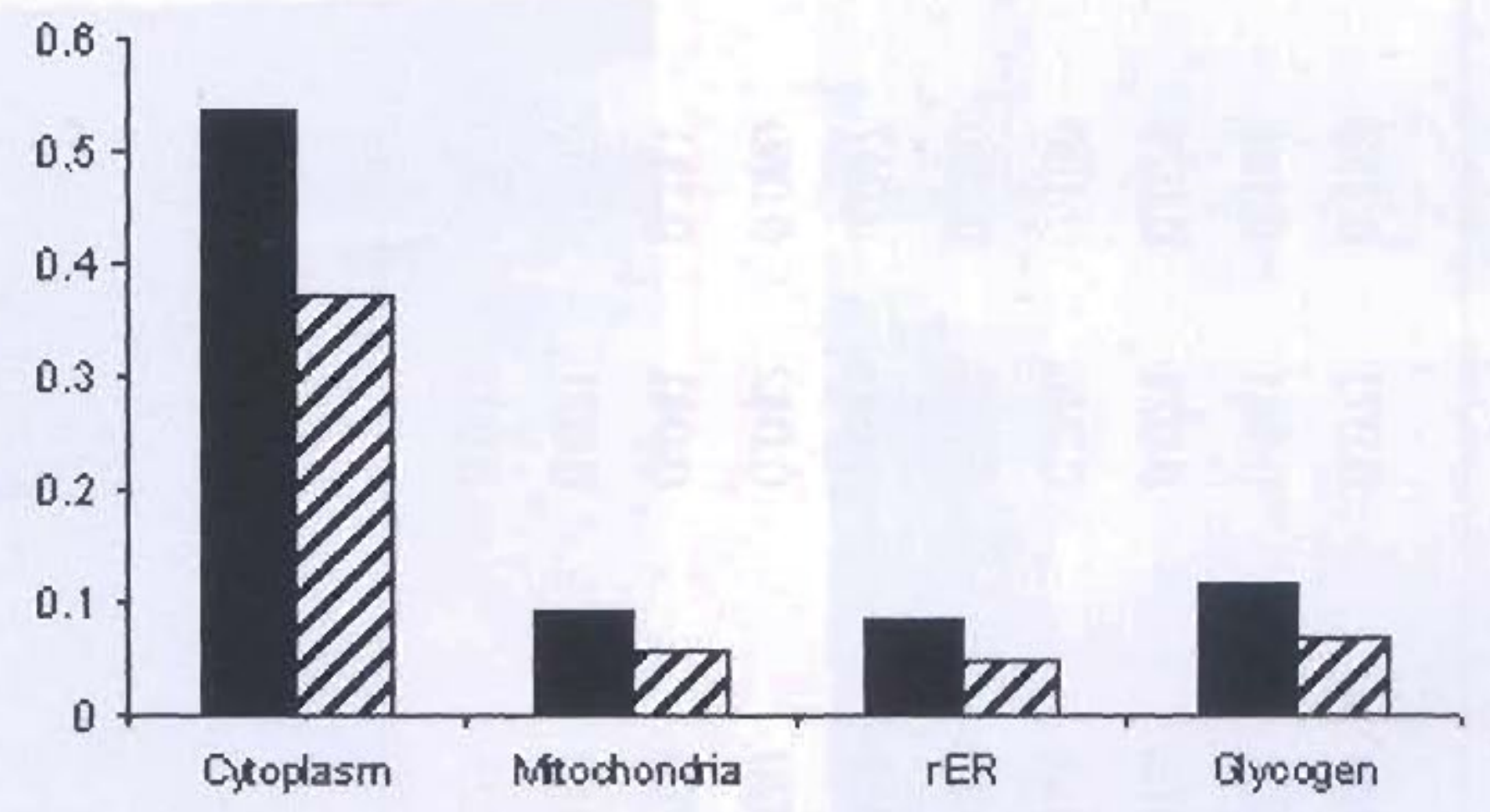

Figure 3. Comparison of volume fractions of endothelial cell cytoplasm and organelles of terminal capillaries of placentae of hypertensive $(-)$ and non-hypertensive $(\mathbb{S})$ mothers.

\section{Discussion}

Ultrastructural changes observed in the present study of placentae of hypertensive and non-hypertensive mothers highlights the many different features that may occur not only in different placentae and different regions but often in adjacent chorionic villi. The results of the present study are in agreement with those of previous studies regarding the characteristics of the different types of organelles present in the capillaries of endothelial cells (14). The presence of numerous mitochondria, rough endoplasmic reticulum, glycogen deposits and granules indicate that the endothelial cells are metabolically active.

It is well known that the placenta is subjected to some degree of ischaemia leading to hypoxia when a significant decrease in utero-placental blood flow occurs in preeclampsia $(15,16)$. Since the hypoxic effect on the syncytium is a direct effect, the syncytium 
undergoes degeneration with a decrease in the number and size of the mitochondria and rough endoplasmic reticulum which may lead to a reduction in the energy available for active transport mechanisms. Vacuolation and altered architectural pattern of mitochondria and thickening of the capillary basement membrane shown in endothelial cells of terminal capillaries of placentae in pre-eclampsia suggest that hypoxia affects the fetal capillaries as well. These changes occur despite the indirect nature of the hypoxic insult. Changes of mitochondrial architecture and vacuolations in endothelial cells and pericytes have been described, even in the systemic capillaries exposed to hypoxia (17). With the damage to mitochondria generation of adenosine triphosphate (ATP) will be reduced. Up to a certain limit cells have the ability to counteract the reduced synthesis of ATP by synthesizing more mitochondria (18). This results in an increase in the volume fraction of mitochondria in capillary endothelial cells of placentae of hypertensive pregnancies when compared to placentae from nonhypertensive pregnancies. This is in keeping with the findings of our study. The damage and compensatory increase in the number and the size of the mitochondria are associated with a significant increase in the volume fraction of rough endoplasmic reticulum in placental fetal capillary endothelial cells of the hypertensive group. It could also be anticipated that in hypertensive pregnancies the capillary endothelial cells would need more energy for their enhanced synthesis, transport and degradation activities when compared to that of capillary endothelial cells of the normotensive group. The fetal blood glucose levels are about two-thirds of those of the mother. Glucose from the mother's blood passes through the placenta by facilitated diffusion. From the 30th gestational week fetal liver becomes efficient and converts glucose into glycogen which is stored in the fetal heart muscle, skeletal muscle and placenta (19). Since the maternal blood flow to the placenta is reduced in hypertension, the placenta may be absorbing the maximum amount of glucose from maternal blood and the excess over immediate metabolic requirements may be converted into glycogen and stored in the placenta. The capacity of endothelial cell cytoplasm is increased perhaps to accommodate excessive degradation of damaged organelles, increased synthesis of new organelles, and to store excess glycogen. However, by using modern and more accurate methods to measure the cytoplasmic volume and organelles, more conclusive findings can be obtained. In this study, we have demonstrated that the mitochondria, rough endoplasmic reticulum and glycogen deposits were increased in endothelial cells of terminal capillaries of placentae of hypertensive mothers in comparison with those of non- hypertensive mothers.

\section{References}

1. Heinrich D., Metz J., Raviola E., Forssmann W.G. Ultrastructure of perfusion fixed fetal capillaries in the human placenta. Cell and Tissue. Research 1976; 172: 157-169.

2. Kaufmann P., Schroeder H., Leichtweis H.P. Fluid shift across the placenta. Feto maternal transfer of horse radish peroxidase in the guinea- pig. Placenta 1982; 3: 339-348.

3. Brosens I., Dixon H.G., Robertson W.B. Fetal growth retardation and the arteries of the placental bed. British Journal of Obstetrics and Gynaecology 1977; 84: 656-663. 
4. Khong T.Y., De Wolf F, Robertson W.B., Brosens I. Inadequate maternal vascular response to placentation in pregnancies complicated by pre-eclampsia and by small for gestational age infants. British Journal of Obstetrics and Gynaecology 1986; 93: 1049-1059.

5. Robertson W.B., Brosens I., Dixon H.G. The pathological response of the vessels of the placental bed to hypertensive pregnancy. Journal of Pathology and Bacteriology1967; 93: 581-592.

6. Jones C.J.P, Fox H. An ultrastructural and ultrahistochemical study of the human placenta in maternal preeclampsia. Placenta 1980; 1: 61-76.

7. Smarason A.K., Sargent I.L., Starkey P.M., Redmann C.W.C.The effect of placental syncytiotrophoblast microvillus membranes from normal and preeclamptic women on the growth of endothelial cells in vitro. British Journal of Obstetrics and Gynaecology 1993; 100: 943-949.

8. Rodgers G.M., Taylor R.N., Roberts J.M. Pre-eclampsia is associated with a serum factor cytotoxic to human endothelial cells. American Journal of Obstetrics and Gynaecology1988; 159: 908-914.

9. Roberts J. M. Endothelial dysfunction' in pre-eclampsia. Seminars in Reproductive Endocrinology 1998; 16: 5-15.

10. Walsh S.W. Pre-eclampsia: An imbalance in placental prostacyclin and thromboxane production. American Journal of Obstetrics and Gynaecology 1985; 152: 335-340.

11. Roberts J.M., Taylor R.N., Musci T.J., Rodgers G.M., Hubel C.A., McLaughlin
M.K. Pre-eclampsia: An endothelial cel disorder. American Journal of Obstetrics and Gynaecology1989; 16 1200-1204.

12. Weibel E.R., Gnani H.R. Improvements in efficiency of stereologic methods in electron microscopic cytology. In: Bocciarelli D.S(eds) Electron Microscopy 1(Ed. by Fourth European Regional Conference. Rome,1968; 1 601-602.

13. Weibel E.R. Stereological principles of morphometry in electron microscopic cytology. Internal Review Cytology 1969; 26: 235-302.

14. Jones C.J.P., Fox H. Ultrastructure of the normal human placenta. Electron microscopy Review 1991; 4: 129-178.

15. Browne J.C.M., Veall N.The materna placental blood flow in normotensive and hypertensive women. Journal of Obstetrics and Gynaecology British Empire 1953; 60: 141-147.

16. Dixon H.G., Browne J.C.M., Davey D.A Choriodecidual and myometrial blood flow. Lancet 1963; 2: 369-373.

17. Binowsky A., Remis T., Benushka J., Massarora M. Ultrastructural changes in the brain capillaries after hypoxia. Folia Morphologia 1990; 38: 236-240.

18. Egil Fosslien. Mitochondrial medicine, molecular pathology of defective oxidative phosphorylation. Annals of Clinical and Laboratory Science. 2001; 31: 25-67.

19. Derek Llewellyn-Jones. Fetoplacental unit. Fundamentals of Obstetrics and Gynaecology, London: Faber and Faber. 1990; 1: 22-30. 